\title{
Additional Treatment with Low-Molecular-Weight Heparin Provides a Better Patient Outcome for Neonatal Pulmonary Hemorrhage with Unfractionated Heparin Treatment
}

\author{
Youmin Zheng, ${ }^{1}$ Lingling Chen, ${ }^{1}$ Lingzi Zhang, ${ }^{1}$ and Yongxia Liu $\mathbb{D}^{2}$ \\ ${ }^{1}$ Taizhou Hospital of Zhejiang Province, China \\ ${ }^{2}$ Department of Blood Transfusion, Yanan University Affiliated Hospital, No. 43 North Street, Baota District, Yanan City, \\ Shanxi Province 716000, China
}

Correspondence should be addressed to Yongxia Liu; liuyongxia9303@163.com

Received 26 March 2021; Accepted 24 April 2021; Published 11 May 2021

Academic Editor: Songwen Tan

Copyright ( 2021 Youmin Zheng et al. This is an open access article distributed under the Creative Commons Attribution License, which permits unrestricted use, distribution, and reproduction in any medium, provided the original work is properly cited.

Pulmonary hemorrhage occurring in preterm newborns is a catastrophic event and is significantly associated with neonatal deaths. Low-weight-molecular heparin is a medical agent usually used as anticoagulants during pregnancy and has the advantages of good absorption, long half-life, and high bioavailability. This study evaluated the pulmonary function and coagulation function in neonates with pulmonary hemorrhage following intravenous drip of low-molecular-weight heparin and the effects of lowmolecular-weight heparin on serum prealbumin and retinol-binding protein levels. A total of 96 neonates with pulmonary hemorrhage were included as study subjects and arranged into the control group and the observation group, 48 per group, based on intravenous drip of unfractionated heparin with or without low-molecular-weight heparin. The neonates receiving intravenous drip of unfractionated heparin and low-molecular-weight heparin exhibited elevated partial pressure of oxygen $\left(\mathrm{PaO}_{2}\right)$ concomitant with declined partial pressure of carbon $\left(\mathrm{PaCO}_{2}\right)$ compared to those receiving unfractionated heparin treatment alone. With regard to pulmonary function, neonates receiving combined treatment of unfractionated heparin and low-molecular-weight heparin displayed increased forced expiratory volume in the first second (FEV1), FEV1/forced expiratory vital capacity $(\mathrm{FVC})$, and peak expiratory flow $(\mathrm{PEF})(P<0.05)$ when comparable to neonates receiving unfractionated heparin treatment alone. As for coagulation function, neonates with pulmonary hemorrhage had decreased activated partial thromboplastin time (APTT), prothrombin time (PT), thromboplastin time (TT), and fibrinogen (FIB) after treatment. Expectedly, these decreases were more significantly in neonates undergoing unfractionated heparin coupled with low-molecularweight heparin $(P<0.05)$. The control group was given unfractionated heparin, and the observation group was given unfractionated heparin coupled with low-molecular-weight heparin. In addition to pulmonary function and coagulation function, it was also observed that neonates undergoing unfractionated heparin coupled with low-molecular-weight heparin exhibited higher serum levels of serum prealbumin and retinol-binding protein than those treated with unfractionated heparin alone. Finally, higher recovery rate and lower incidence rate of complications, such as pulmonary infection, intracranial hemorrhage, and respiratory distress, were found in the observation group than the control group $(P<0.05)$. In conclusion, additional treatment with low-molecular-weight heparin could provide a better patient outcome for neonatal pulmonary hemorrhage with unfractionated heparin treatment, as it could notably improve pulmonary function and coagulation function and reduce the incidence of complications.

\section{Introduction}

Pulmonary hemorrhage represent a life-threatening event that shows an approximate incidence of $0.1 \%$ in all infants, while the incidence rate reaches to $5 \%-11 \%$ in newborns who are preterm or suffer intrauterine growth restriction [1]. Preterm infants usually experience pulmonary hemorrhage within 72 hours after birth, leading to significant morbidity and mortality and long-term neurological sequelae [2]. The occurrence of neonatal pulmonary hemorrhage is 
attributed to several factors including low body weight, premature delivery, intrauterine growth restriction, coagulation dysfunction, infection, the administration of exogenous surfactant, intubation in delivery room, and previous use of blood components [3-5]. Interestingly, the phenomenon that pulmonary hemorrhage follows a neurological insult has been termed as neurogenic pulmonary hemorrhagic edema, indicating the relationship between pulmonary hemorrhage and neonatal neurological disease [6]. The histologic characteristics of the lung in the context of pulmonary hemorrhage remain to be characterized, and it seems that pulmonary maldevelopment such as pulmonary hypoplasia and presence of intra-acinar arterioles may contribute to pulmonary hemorrhage. So far, mechanical ventilation, medication, and circulatory management have been performed treating neonatal pulmonary hemorrhage [7-9], but the mortality rate of the children is still high.

Heparin is a polysaccharide activator of antithrombin by producing protease interaction exosites for blood clotting proteinases, thus promoting the inactivation of thrombin. Nonfractionated heparin has a mixture of polysaccharides with molecular weight from 3,000 to 30,000 Daltons [10, 11]. The administration of heparin has been reported to reduce the risk of developing intraventricular hemorrhage and parenchymal venous infarct which is a condition complicating intraventricular hemorrhage [12]. Low-weightmolecular heparin is a medical agents usually used as anticoagulants during pregnancy and has the advantages of good absorption, long half-life, high bioavailability, and low incidence of adverse reactions $[13,14]$. The purpose of this study is to analyze the effect of low-molecular-weight heparin on pulmonary function and coagulation function of newborn with pulmonary hemorrhage and additional influence on expressions of serum prealbumin and retinol-binding protein, so as to provide reference for clinical treatment.

\section{Material and Method}

2.1. Inclusion and Exclusion Criteria. This retrospective study received the approval of Ethics Committee of our hospital, and the informed consent was obtained from each guardian. Eligible newborns with pulmonary hemorrhage should meet the following inclusion criteria: (a) in accordance with the "diagnosis and treatment of neonatal pulmonary hemorrhage" issued by Chinese Medical Association; 9b) the platelet level was less than $80 \times 10^{9} / \mathrm{L}$; (c) the mother of neonate the did not use drugs related to fibrinolysis and coagulation during prenatal and delivery; (d) all the guardians of the neonate implemented informed consent. The newborns with pulmonary hemorrhage who died within 24 hours after admission or whose family members gave up treatment, complicated by congenital heart disease and abnormal liver and kidney function, congenital genetic diseases, hyperbilirubinemia, severe intrauterine infection, coagulation dysfunction, or immune function diseases, and stayed in coma after resuscitation treatment with low Apgar score (PMID: 3183690) (no less than 3) should be excluded.
2.2. Eligible Study Subjects. A number of 96 neonates with pulmonary hemorrhage in our hospital from December 2016 to December 2018 were analyzed and divided into the observation group with 48 cases and the control group with 48 cases according to different treatments. The control group involved 28 males and 20 females, with age ranging from 1 to 21 days, an average of $7.59 \pm 0.51$ days, weighing from 2011 to $4510 \mathrm{~g}$, an average of $3520 \pm 720 \mathrm{~g}$; with Apgar scores ranging from 5 to 10 points, an average of $7.04 \pm 1.35$ points; with onset time ranging from 30 minutes to 24 days after birth, an average of $11.28 \pm 3.54$ days. There were 25 premature infants, 13 full-term infants, and 10 late born infants among them. There were 30 males and 18 females in observation group involving 24 cases of premature infants, 13 cases of full-term infants, and 11 cases of late birth, with aging from 1 to 24 days, an average age of $7.39 \pm 0.48$ days, weighing from 2046 to $4571 \mathrm{~g}$, an average weight of $3582 \pm 697 \mathrm{~g}$; with Apgar scores from 5 to 10 points, an average of $7.10 \pm 1.29$ points; with onset time ranging from 40 minutes to 22 days after birth, an average of onset time $11.15 \pm 3.45$ days. The two groups were comparable $(P>0.05)$.

2.3. Management Protocols. All newborns with pulmonary hemorrhage were given regular treatments including antishock measures, anti-infection, better fluid, and electrolyte balance. Afterwards, they underwent orotracheal intubation by using the ventilator (Stephanie, Germany) with inspired oxygen (FiO2) 0.6-1.0, mean airway pressure (MAP) 0.78$1.18 \mathrm{kPa}$, inspiratory time $(\mathrm{Ti}) 33 \%$, respiration rate $(\mathrm{RR}) 8$ $12 \mathrm{~Hz}$, and amplitude 3-5 (oscillating pressure 1.96-4.90). Parameters were changed according to the blood gas value, and the $\mathrm{pH}$ value of blood gas, partial pressure of oxygen $\left(\mathrm{PaO}_{2}\right)$, partial pressure of carbon $\left(\mathrm{PaCO}_{2}\right)$ were, respectively, maintained at 7.4-7.5, 60-80 mmHg, and 35$55 \mathrm{mmHg}$. When fraction of inspired oxygen $\left(\mathrm{FiO}_{2}\right)$ was no more than $40 \%$, and mean airway pressure (MAP) was no more than $0.78 \mathrm{kPa}$, it can be transferred to synchronized intermittent mandatory ventilation (SIMV), and continuous positive airway pressure (CPAP) was given after ventilator removed. The control group was treated with unfractionated heparin $(6 \mu \mathrm{g} / \mathrm{kg}$, body weight, No. 21071115, Hainan Changshi Medicine Co., Ltd., China) injected into vein once every 6 hours, and the drug was withdrew after the activated partial thromboplastin time (APTT), prothrombin time (PT), fibrinogen (FIB), and thrombin time (TT) recovered, respectively, to $25-37 \mathrm{~s}, 11-14 \mathrm{~s}, 2-4 \mathrm{~g} / \mathrm{L}$, and $12-16 \mathrm{~s}$. As for the observation group, on the basis of the treatment in the control group, were additionally treated with lowmolecular-weight heparin $(40 \mu \mathrm{g} / \mathrm{kg}$, body weight, No. 21060805, Jilin Huakang Pharmaceutical Co., Ltd., China) dripped into vein twice a day for preventive treatment, and the withdrawal criteria were consistent with that of the control group.

2.4. Outcomes. The value of $\mathrm{PaO}_{2}$ and $\mathrm{PaCO}_{2}$ were, respectively, recorded before, $6 \mathrm{~h}$, and $24 \mathrm{~h}$ after treatment. The changes of the first second forced expiratory volume (FEV1), FEV1/forced vital capacity (FVC), and peak expiratory flow $(\mathrm{PEF})$ were analyzed by pulmonary function tester 
(CHESTAT-8800, Japan). Blood sample ( $2 \mathrm{~mL}$ ) was collected from each fasting patient before and after treatment and placed in tube with the presence of 0.109 mol sodium citrate with ratio $1: 9$. APTT, PT, TT, and FIB were measured by automatic blood coagulation analyzer (CA15000, SYSMEX, Japan). The levels of prealbumin and retinol-binding protein before and after treatment were detected by automatic biochemical analyzer (MODULE P800, Roche, Switzerland) using corresponding kits (Sichuan Maccura Biotechnology Co., Ltd., China).

2.5. Recovery Criteria of Pulmonary Hemorrhage. The blood gas value and ventilator parameters returned to normal, and there was no blood aspirated from trachea. Bilateral pulmonary rales disappeared or significantly reduced, and clinical symptoms disappeared or significantly improved.

2.6. Statistical Methods. SPSS21.0 statistical software was used for data analysis. The counting data were expressed as percentage (\%) and detected by chi-square test; the measurement data were described as mean \pm standard deviation and analyzed by $t$ test and one-way and two-way analysis of variance (ANOVA). With $\alpha=0.05$ as the test level, the $P$ value of less than 0.05 indicates that the difference was statistically significant.

\section{Results}

3.1. Additional Treatment with Low-Molecular-Weight Heparin Maintained Arterial Partial Pressures of Neonates with Pulmonary Hemorrhage. $\mathrm{PaCO}_{2}$ and $\mathrm{PaO}_{2}$ are an essential component of patient care. We first examined $\mathrm{PaCO}_{2}$ and $\mathrm{PaO}_{2}$ of each patient in the observation and control group before, $6 \mathrm{~h}$, and $24 \mathrm{~h}$ after treatment. It was found that the $\mathrm{PaO}_{2}$ was notably increased, and $\mathrm{PaCO}_{2}$ was descended in both groups after intravenous drip of unfractionated heparin and low-molecular-weight heparin $(P<0.05)$. The newborns with pulmonary hemorrhage undergoing unfractionated heparin coupled with low-molecular-weight heparin exhibited elevated $\mathrm{PaO}_{2}$ concomitant with declined $\mathrm{PaCO}_{2}$ compared with those receiving unfractionated heparin treatment alone at indicated time points $(6 \mathrm{~h}$ and $24 \mathrm{~h}$ after treatment $)(P<0.05)$. Additionally, the elevation of $\mathrm{PaO}_{2}$ and the reduction of $\mathrm{PaCO}_{2}$ were greater in the observation group when comparable to the control group $(P<0.05$, Table 1 and Figure 1).

3.2. Additional Treatment with Low-Molecular-Weight Heparin Restored Pulmonary Function of Neonates with Pulmonary Hemorrhage. Pulmonary function testing is usually performed to diagnose many categories of pulmonary disease. We next evaluated the FEV1, FEV1/FVC, and PEF to reflect the pulmonary function of neonates with pulmonary hemorrhage. No remarkable difference was observed between the observation and control group before treatment $(P>0.05)$. After unfractionated heparin treatment with or without additional low-molecular-weight heparin treatment, FEV1, FEV1/FVC, and PEF were all increased in the two groups $(P<0.05)$. The newborns with pulmonary hemorrhage undergoing unfractionated heparin coupled with low- molecular-weight heparin exhibited elevated FEV1, FEV1/FVC, and PEF when comparable to those receiving unfractionated heparin treatment alone $(P<0.05$, Table 2$)$.

3.3. Additional Treatment with Low-Molecular-Weight Heparin Improved Coagulation Function of Neonates with Pulmonary Hemorrhage. Pulmonary hemorrhage is a coagulation disorder. In this part, the anticoagulation effects of unfractionated heparin treatment with or without additional low-molecular-weight heparin treatment on pulmonary hemorrhage were evaluated. As shown in Table 3, before treatment, the observation and control groups were comparable as no significance existed in coagulation parameters $(P>0.05)$. After unfractionated heparin treatment with or without additional low-molecular-weight heparin treatment, APTT, PT, TT and FIB were all remarkably reduced in the two groups $(P<0.05)$. These reductions were more significant in the newborns with pulmonary hemorrhage undergoing unfractionated heparin coupled with low-molecularweight heparin than those receiving unfractionated heparin treatment alone $(P<0.05)$.

3.4. Additional Treatment with Low-Molecular-Weight Heparin Increased Prealbumin and Retinol-Binding Protein Levels in Neonates with Pulmonary Hemorrhage. Prealbumin and retinol-binding protein belong to hepatic secretory proteins that are markers of visceral protein stores and used as indicators of nutritional status. The serum levels of prealbumin and retinol-binding protein showed little difference between the two groups before unfractionated heparin treatment with or without additional low-molecular-weight heparin treatment $(P>0.05)$. After treatment, there were significant increases in the serum levels of prealbumin and retinol-binding protein in the two groups $(P<0.05)$. The newborns with pulmonary hemorrhage undergoing unfractionated heparin coupled with low-molecular-weight heparin exhibited elevated serum levels of prealbumin and retinolbinding protein compared with those receiving unfractionated heparin treatment alone $(P<0.05$, Figure 2$)$.

3.5. Efficacy and Safety of Additional Treatment with LowMolecular-Weight Heparin for Neonates with Pulmonary Hemorrhage. Finally, we calculated the recovery rate and incidence rate of complications, such as pulmonary infection, intracranial hemorrhage, and respiratory distress to evaluate the efficacy and safety of additional treatment with lowmolecular-weight heparin for neonates with pulmonary hemorrhage. There were 35 cured neonates out of 48 cases in the control group with $72.92 \%$ cure rate and involved 3 cases of pulmonary infection, 2 cases of intracranial hemorrhage, 1 case of respiratory distress out of 48 cases with $12.50 \%$ complication rate; there were 44 cured neonates out of 48 cases in the observation group with $91.67 \%$ cure rate and contained 1 case of pulmonary infection and 1 case of intracranial hemorrhage out of 48 cases with $4.17 \%$ complication rate, suggesting that the cure rate and complication rate improved significantly in the observation group compared with the control group $(P<0.05$, Figure 3$)$. 
TABle 1: The $\mathrm{PaCO}_{2}$ and $\mathrm{PaO}_{2}$ of each patient in the observation and control group before, $6 \mathrm{~h}$, and $24 \mathrm{~h}$ after unfractionated heparin treatment with or without additional low-molecular-weight heparin treatment.

\begin{tabular}{lccc}
\hline Group & Time & PaO2 $(\mathrm{mmHg})$ & PaCO2 $(\mathrm{mmHg})$ \\
\hline & Before treatment & $50.62 \pm 5.01$ & $59.39 \pm 4.74$ \\
Control group $(n=48)$ & $6 \mathrm{~h}$ after treatment & $56.46 \pm 6.16$ & $50.16 \pm 5.24$ \\
& 24 h after treatment & $72.41 \pm 4.56$ & $42.51 \pm 3.11$ \\
$F$ & & 31.410 & 16.120 \\
$P$ & Before treatment & 0.001 & 0.001 \\
& 6h after treatment & $50.171 \pm 5.89$ & $59.31 \pm 4.77$ \\
Observation group $(n=48)$ & 24 h after treatment & $62.72 \pm 4.51$ & $42.29 \pm 4.26$ \\
& & $84.28 \pm 3.71$ & $37.01 \pm 2.75$ \\
$P$ & & 57.740 & 32.630 \\
\hline
\end{tabular}

PaO2: partial pressure of oxygen; PaCO2: artery partial pressure of carbon.

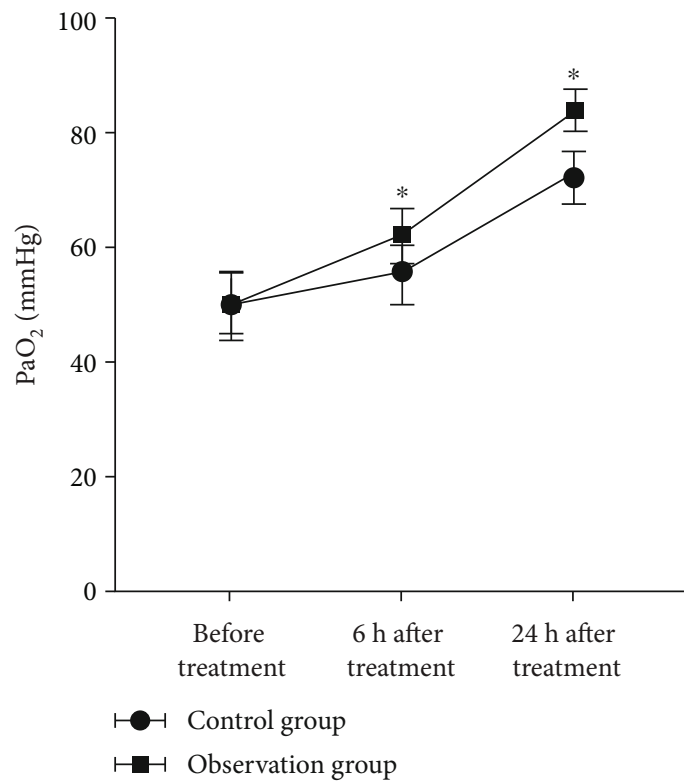

(a)

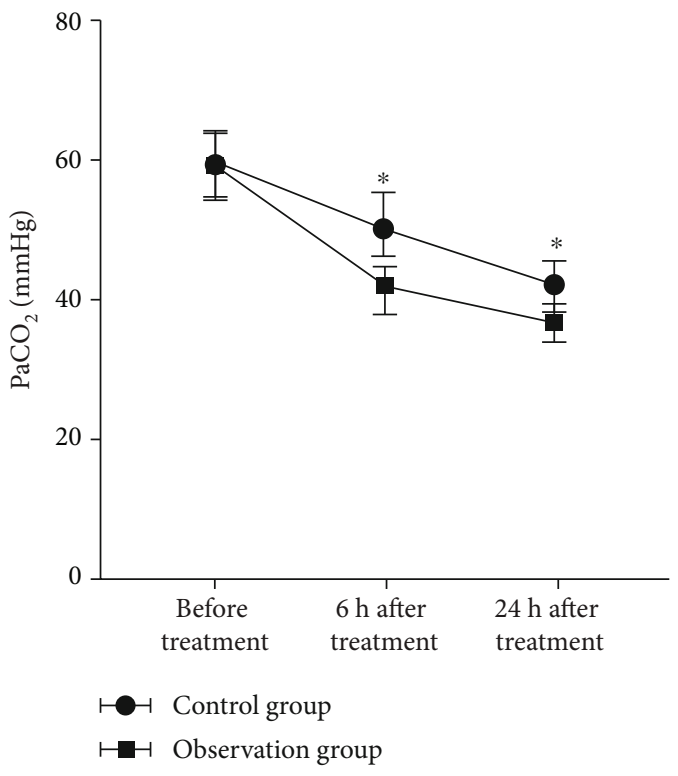

(b)

Figure 1: Alternations of $\mathrm{PaCO}_{2}$ and $\mathrm{PaO}_{2}$ of neonates with pulmonary hemorrhage before, $6 \mathrm{~h}$, and $24 \mathrm{~h}$ after intravenous drip of unfractionated heparin with or without low-molecular-weight heparin treatment. ${ }^{*}$ indicates $P<0.05$ compared with the control group at indicated time points after treatment.

\section{Discussion}

Heparin is an anticoagulant for the prevention and treatment of venous thrombosis [15]. Low-molecular-weight heparin prepared from heparin has different pharmacokinetic and pharmacodynamics characteristics from unfractionated heparin, and it can reduce capillary permeability, improve local edema, and promote smooth muscle contraction and local hemostasis [16]. At the same time, low-molecular-weight heparin can significantly improve the vasoconstriction and oxygen supply in neonate [17].
The alveolar elastic fibrous tissue and bronchial wall fiber development of neonate are dysplasia, and alveolar surfactant is less, prone to alveolar and bronchial occlusion, which leading to hypoxia and microvascular damage [18]. Neonates with lung dysplasia have more blood vessels with less alveolar tissue, easy to cause bleeding phenomenon [19]. The increase of capillary pressure in alveolar wall caused by many factors leads to pulmonary hemorrhagic disease. Pulmonary hemorrhage is an important cause of perinatal death and complications [20]. Low-molecular-weight heparin plays a positive role in improving lung function of patients with lung disease. 
TABLE 2: The FEV1, FEV1/FVC, and PEF of each patient in the observation and control group before and after unfractionated heparin treatment with or without additional low-molecular-weight heparin treatment.

\begin{tabular}{|c|c|c|c|c|}
\hline Group & Time & FEV1 (L) & FEV1/FVC (\%) & $\mathrm{PEF}(\mathrm{L} / \mathrm{s})$ \\
\hline \multirow{2}{*}{ Control group $(n=48)$} & Before treatment & $1.31 \pm 0.36$ & $49.6 \pm 7.44$ & $5.90 \pm 0.96$ \\
\hline & After treatment & $2.58 \pm 0.48$ & $58.6 \pm 8.65$ & $7.11 \pm 1.21$ \\
\hline$t$ & & 2.586 & 5.172 & 3.857 \\
\hline$P$ & & 0.012 & 0.007 & 0.014 \\
\hline \multirow{2}{*}{ Observation group $(n=48)$} & Before treatment & $1.30 \pm 0.41$ & $49.2 \pm 6.48$ & $5.84 \pm 0.78$ \\
\hline & After treatment & $3.15 \pm 0.52^{*}$ & $66.9 \pm 7.44^{*}$ & $8.85 \pm 1.33^{*}$ \\
\hline$t$ & & 3.125 & 8.635 & 5.173 \\
\hline$P$ & & 0.021 & 0.001 & 0.001 \\
\hline
\end{tabular}

The symbol of * indicates $P<0.05$ compared with the control group; FEV1: the first second forced expiratory volume; FVC: forced vital capacity; PEF: peak expiratory flow.

TABLE 3: The changes of coagulation parameters function of each patient in the observation and control group before and after unfractionated heparin treatment with or without additional low-molecular-weight heparin treatment.

\begin{tabular}{|c|c|c|c|c|c|}
\hline Group & Time & $\operatorname{APTT}(\mathrm{s})$ & $\mathrm{PT}(\mathrm{s})$ & TT (s) & FIB $(\mathrm{g} / \mathrm{L})$ \\
\hline \multirow{2}{*}{ Control group $(n=48)$} & Before treatment & $82.3 \pm 8.52$ & $19.3 \pm 2.69$ & $20.8 \pm 2.76$ & $5.24 \pm 0.83$ \\
\hline & After treatment & $66.6 \pm 7.25$ & $14.1 \pm 2.85$ & $15.6 \pm 2.63$ & $3.31 \pm 0.79$ \\
\hline$t$ & & 10.176 & 6.175 & 5.746 & 3.963 \\
\hline$P$ & & 0.001 & 0.001 & 0.004 & 0.024 \\
\hline \multirow{2}{*}{ Observation group $(n=48)$} & Before treatment & $83.0 \pm 9.77$ & $19.6 \pm 3.10$ & $21.3 \pm 3.05$ & $5.14 \pm 0.79$ \\
\hline & After treatment & $50.5 \pm 8.35^{*}$ & $10.2 \pm 1.24^{*}$ & $12.3 \pm 1.25^{*}$ & $2.57 \pm 0.76^{*}$ \\
\hline$t$ & & 21.084 & 8.173 & 6.712 & 4.183 \\
\hline$P$ & & 0.001 & 0.001 & 0.001 & 0.016 \\
\hline
\end{tabular}

The symbol of * indicates $P<0.05$ compared with the control group; APTT: activated partial thromboplastin time; PT: prothrombin time; TT: thrombin time; FIB: fibrinogen.

Nasal continuous positive airway pressure (NCPAP) combined with low-molecular-weight heparin in the treatment of severe pneumonia has a good effect, which is helpful to improve the lung function and blood oxygen level of patients [21]. Low-molecular-weight heparin combined with acetylcysteine in the treatment of IIPs can improve the lung function of patients more effectively than single drug treatment. On the basis of conventional treatment, low-molecularweight heparin combined with acetylcysteine in the treatment of IIPs can improve lung function more effectively than single drug treatment. On the basis of conventional treatment, the use of low-dose low-molecular-weight heparin calcium in the treatment of chronic obstructive pulmonary disease can significantly improve the total effective rate and blood gas index. The effect of rivaroxaban in the treatment of elderly patients with acute exacerbation of chronic obstructive pulmonary disease (AECOPD) is better than that of low-molecular-weight heparin calcium injection, which may be related to the age of patients. In the future research, we will analyze the patients with different ages separately to provide reference for more reasonable clinical treatment. On the basis of conventional treatment, increasing the use of low-molecular-weight heparin can improve the treatment effect of acute exacerbation of chronic obstructive pulmonary disease, and low-molecular-weight heparin combined with budesonide in the treatment of severe pneumonia in children can help to improve tidal breathing pulmonary function and accelerate the disappearance of symptoms and signs. This study also found that FEV1, FEV1/FVC, and peak expiratory flow of the two groups were significantly increased after treatment, suggesting that low-molecular-weight heparin can significantly improve pulmonary function and coagulation function in neonate with pulmonary hemorrhage. In the future study, we will further analyze the changes of blood gas indexes of the two groups after treatment, so as to provide reference for more reasonable clinical treatment.

Organ system development of neonate is not perfect, and they are prone to get infection, shock, even disseminated intravascular coagulation, and organ failure which cause death. Incomplete development of neonatal coagulation mechanism is the main cause of neonatal pulmonary hemorrhage death. At the same time, due to the physiological protection of the newborn, the coagulation function of the newborn is maintained in a low activity state. Therefore, improving coagulation dysfunction and hemostasis is an important idea for clinical treatment. APTT, PT, TT, and FIB are sensitive indicators of coagulation system [22]. Low-molecular-weight heparin can play a role in the 


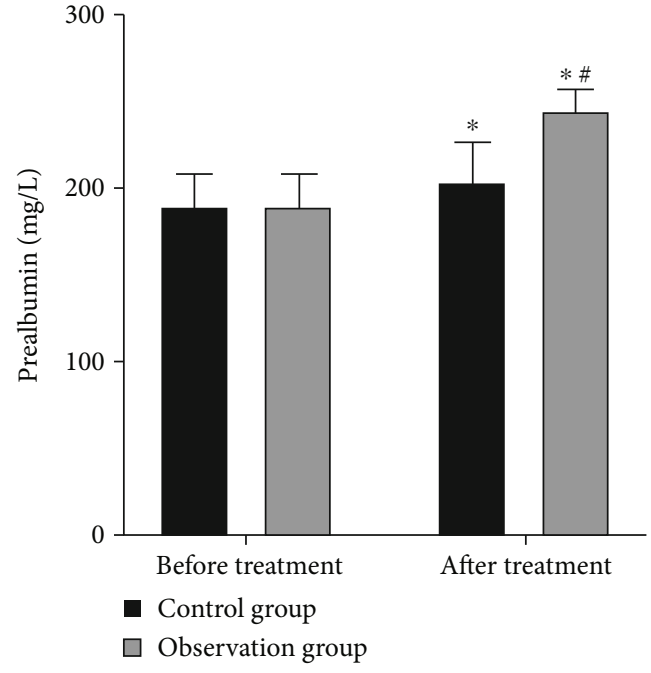

(a)

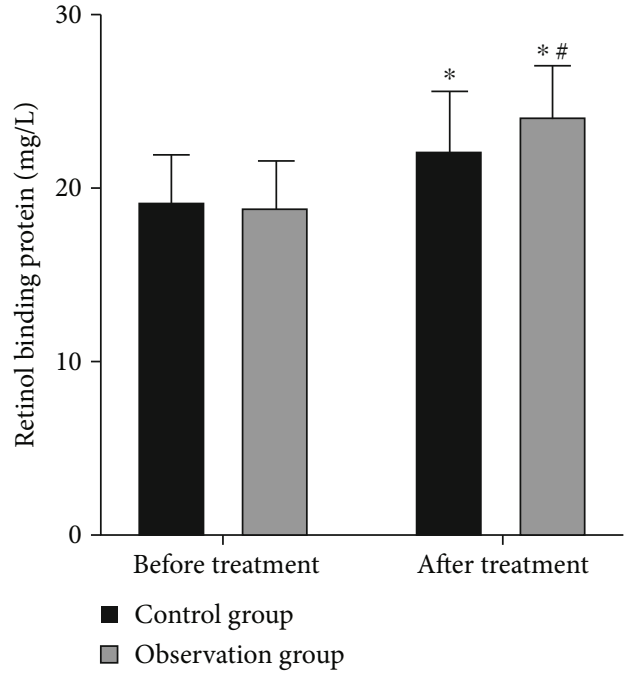

(b)

FIgURE 2: The serum levels of prealbumin and retinol-binding protein in neonates with pulmonary hemorrhage before and after intravenous drip of unfractionated heparin with or without low-molecular-weight heparin treatment. ${ }^{*}$ indicates $P<0.05$ after treatment. ${ }^{\#}$ indicates $P$ $<0.05$ compared with the control group.

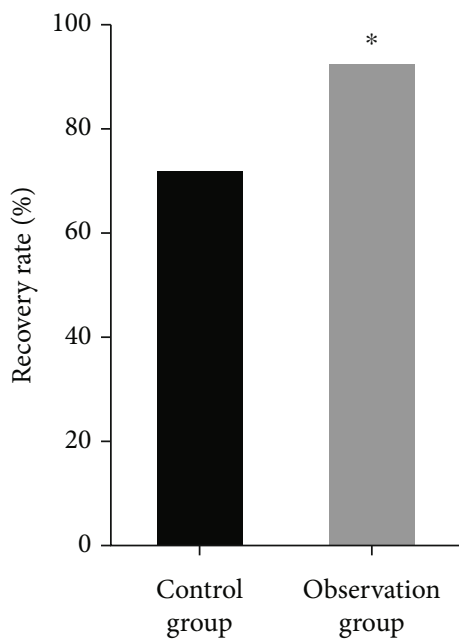

(a)

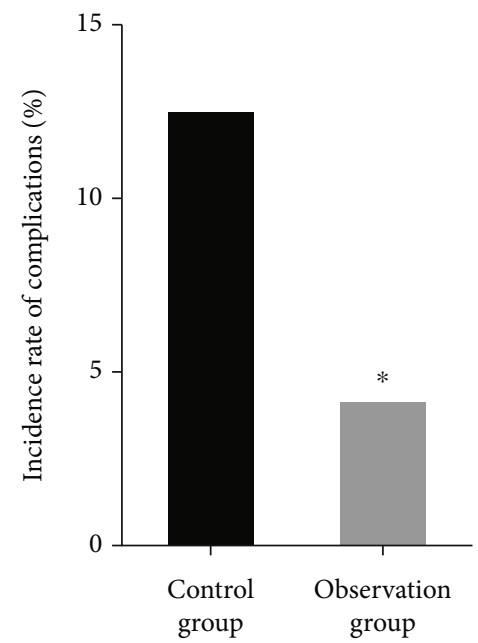

(b)

FIGURE 3: The recovery rate and incidence rate of complications after intravenous drip of unfractionated heparin with or without lowmolecular-weight heparin treatment. * indicates $P<0.05$ compared with the control group.

treatment of venous thrombosis by regulating the abnormal levels of the above factors. Low-molecular-weight heparin combined with prednisone can effectively improve the renal function of children with nephrotic syndrome, but has no obvious adverse effect on blood coagulation. Fasudil hydrochloride combined with low-molecular-weight heparin calcium can affect APTT, PT, TT, and FIB concentrations, improve blood hypercoagulability in patients with ischemic stroke, and improve the clinical therapeutic effect of ischemic stroke. This study also found that the pulmonary function indexes of the two groups were significantly improved after treatment, and the coagulation function indexes such as activated partial thromboplastin time, prothrombin time, and the level of coagulogenic enzyme phase were significantly decreased, suggesting that the clinical effect of low- molecular-weight heparin and the improvement of pulmonary function in patients with neonatal pulmonary hemorrhage are related to the abnormal coagulation function indexes.

Prealbumin is a thyroxine and retinol-binding protein synthesized by hepatocytes, which belongs to the category of carrier protein [23]. Liver is also an important place for the synthesis of coagulation factors [24], and abnormal or damaged liver function can lead to abnormal expression of coagulation factors and prealbumin. There was significant difference in plasma prealbumin between patients with AECOPD and healthy controls before and after treatment [25]. The detection of plasma prealbumin is helpful to reflect the nutritional status of COPD patients [26]. The decrease of prealbumin in patients with COPD reflects the severity of 
COPD to a certain extent, which can be an important reference index for clinicians to judge the prognosis of COPD. Retinol-binding protein is also a sensitive indicator of nutritional status. In a study reported by Delgado et al., an increased level of retinol-binding protein was found after parenteral support of critically ill infants during short period of intensive care [27].

In conclusion, additional treatment with low-molecularweight heparin could provide a better patient outcome for neonatal pulmonary hemorrhage with unfractionated heparin treatment, as it could notably improve pulmonary function and coagulation function and reduced the incidence of complications. There were many factors influencing newborns, and the number of study subjects included in this study is limited; we will further increase the sample size and observation indicators in future studies and use multicenter comparative study to provide reference for more reasonable clinical treatment.

\section{Data Availability}

The data used to support the findings of this study are included within the article.

\section{Conflicts of Interest}

The authors declare that they have no conflicts of interest.

\section{References}

[1] I. Agarwal and L. M. Ernst, "Perinatal pulmonary hemorrhage: a retrospective autopsy case series," Pediatric and Developmental Pathology, vol. 23, no. 4, pp. 267-273, 2020.

[2] M. Lee, K. Wu, A. Yu et al., "Pulmonary hemorrhage in neonatal respiratory distress syndrome: radiographic evolution, course, complications and long-term clinical outcomes," Journal of Neonatal-Perinatal Medicine, vol. 12, no. 2, pp. 161-171, 2019.

[3] A. Aziz and A. Ohlsson, "Surfactant for pulmonary haemorrhage in neonates," Cochrane Database of Systematic Reviews, vol. 2, article CD005254, 2020.

[4] C. H. Ferreira, F. Carmona, and F. E. Martinez, "Prevalencia, fatores de risco e prognostico associados a hemorragia pulmonar em recem-nascidos," Jornal de Pediatria, vol. 90, no. 3, pp. 316-322, 2014.

[5] R. Orbach, D. Mandel, R. Lubetzky et al., "Pulmonary hemorrhage due to Coxsackievirus B infection-a call to raise suspicion of this important complication as an end-stage of enterovirus sepsis in preterm twin neonates," Journal of Clinical Virology, vol. 82, pp. 41-45, 2016.

[6] A. Lodha, P. S. Shah, and J. Hellmann, "Pulmonary haemorrhage associated with neonatal neurological disease," Heart, Lung \& Circulation, vol. 18, no. 1, pp. 45-48, 2009.

[7] Y. Shi, S. Tang, H. Li, J. Zhao, and F. Pan, "New treatment of neonatal pulmonary hemorrhage with hemocoagulase in addition to mechanical ventilation," Biology of the Neonate, vol. 88 , no. 2, pp. 118-121, 2005.

[8] V. Vobruba, T. Grus, F. Mlejnsky, J. Belohlavek, J. Hridel, and L. Lambert, "Management of severe pulmonary hemorrhage in a neonate on veno-arterial ECMO by the temporary clamping of the endotracheal tube - a case report," Perfusion, vol. 33, no. 1, pp. 77-80, 2018.

[9] B. H. Su, H. Y. Lin, F. K. Huang, M. L. Tsai, and Y. T. Huang, "Circulatory management focusing on preventing intraventricular hemorrhage and pulmonary hemorrhage in preterm infants," Pediatrics and Neonatology, vol. 57, no. 6, pp. 453-462, 2016.

[10] X. Huang, A. R. Rezaie, G. J. Broze Jr., and S. T. Olson, “Heparin is a major activator of the anticoagulant serpin, protein Zdependent protease inhibitor*," The Journal of Biological Chemistry, vol. 286, no. 11, pp. 8740-8751, 2011.

[11] G. Izaguirre, S. Aguila, L. Qi et al., "Conformational activation of antithrombin by heparin involves an altered exosite interaction with protease*," The Journal of Biological Chemistry, vol. 289, no. 49, pp. 34049-34064, 2014.

[12] M. Bruschettini, O. Romantsik, S. Zappettini, R. Banzi, L. A. Ramenghi, and M. G. Calevo, "Heparin for the prevention of intraventricular haemorrhage in preterm infants," Cochrane Database of Systematic Reviews, vol. 5, article CD011718, 2016.

[13] H. Resic, N. Kukavica, V. Sahovic, and F. Masnic, "Different effects of low weight molecular heparin and unfractioned heparin on lipid profile and coagulation at haemodialysis patients," Bosnian Journal of Basic Medical Sciences, vol. 10, no. 1, pp. 56-S62, 2010.

[14] F. E. Canpolat, D. Orhan, S. Yigit, G. Kale, M. Yurdakok, and G. Tekinalp, "The effects of antenatal anticoagulants (lowmolecular-weight heparin and aspirin) on neonatal pulmonary vasculature in rabbits," Pediatric and Developmental Pathology, vol. 13, no. 2, pp. 107-111, 2010.

[15] C. S. Ong, J. A. Marcum, K. J. Zehr, and D. E. Cameron, "A century of heparin," The Annals of Thoracic Surgery, vol. 108, no. 3, pp. 955-958, 2019.

[16] G. Camporese and E. Bernardi, "Low-molecular-weight heparin for thromboprophylaxis," Current Opinion in Pulmonary Medicine, vol. 15, no. 5, pp. 443-454, 2009.

[17] M. A. Rodger, J. C. Gris, J. I. P. de Vries et al., "Low-molecularweight heparin and recurrent placenta-mediated pregnancy complications: a meta-analysis of individual patient data from randomised controlled trials," Lancet, vol. 388, no. 10060, pp. 2629-2641, 2016.

[18] C. D. Baker and S. H. Abman, "Impaired pulmonary vascular development in bronchopulmonary dysplasia," Neonatology, vol. 107, no. 4, pp. 344-351, 2015.

[19] C. Li, X. Li, C. Deng, and C. Guo, "Circulating fibrocytes are increased in neonates with bronchopulmonary dysplasia," PLoS One, vol. 11, no. 6, article e0157181, 2016.

[20] S. Godfrey, "Pulmonary hemorrhage/hemoptysis in children," Pediatric Pulmonology, vol. 37, no. 6, pp. 476-484, 2004.

[21] A. M. De Klerk and R. K. De Klerk, "Nasal continuous positive airway pressure and outcomes of preterm infants," Journal of Paediatrics and Child Health, vol. 37, no. 2, pp. 161-167, 2001.

[22] M. Milos, D. C. Herak, and R. Zadro, "Discrepancies between APTT results determined with different evaluation modes on automated coagulation analyzers," International Journal of Laboratory Hematology, vol. 32, no. 1p2, pp. 33-39, 2010.

[23] Y. Zhang, M. Zhang, X. Yang, and H. Wang, "Significance of retinol binding protein and prealbumin in neonatal nutritional evaluation," Pakistan Journal of Pharmaceutical Sciences, vol. 31, no. 4, pp. 1613-1616, 2018.

[24] H. Stefan and B. Joris, "Measurement of Blood Coagulation Factor Synthesis in Cultures of Human Hepatocytes," Methods in Molecular Biology, vol. 1250, pp. 309-316, 2015. 
[25] S. Arora, K. Madan, A. Mohan, M. Kalaivani, and R. Guleria, "Serum inflammatory markers and nutritional status in patients with stable chronic obstructive pulmonary disease," Lung India, vol. 36, no. 5, pp. 393-398, 2019.

[26] A. Mohan, S. Arora, A. Uniyal et al., "Evaluation of plasma leptin, tumor necrosis factor- $\alpha$, and prealbumin as prognostic biomarkers during clinical recovery from acute exacerbations of chronic obstructive pulmonary disease," Lung India, vol. 34, no. 1, pp. 3-8, 2017.

[27] A. F. Delgado, H. M. Kimura, A. L. Cardoso, D. Uehara, and F. R. Carrazza, "Nutritional follow-up of critically ill infants receiving short term parenteral nutrition," Revista do Hospital das Clinicas; Faculdade de Medicina da Universidade de Sao Paulo, vol. 55, no. 1, pp. 3-8, 2000. 